\title{
SH3KBP1-binding protein 1 prevents epidermal growth factor receptor degradation by the interruption of c-Cbl-CIN85 complex
}

\author{
Lifeng Feng ${ }^{1,2}$, Jin-Tao Wang ${ }^{3}$, Hongchuan $\operatorname{Jin}^{2}$, Kaixian Qian ${ }^{1 *}$ and Jian-Guo Geng ${ }^{3 *}$ \\ ${ }^{1}$ College of Lifescience, Zhejiang University, Hangzhou, Zhejiang, China \\ ${ }^{2}$ Biomedical Research Center, Sir Runrun Shaw Hospital, School of Medicine, Zhejiang University, Hangzhou, Zhejiang, China \\ ${ }^{3}$ Department of Biologic and Materials Sciences, University of Michigan School of Dentistry, Ann Arbor, MI, USA
}

\begin{abstract}
The binding of Cbl-interacting protein of $85 \mathrm{kDa}(\mathrm{CIN} 85)$ to c-Cbl is important to endocytosis and degradation of epidermal growth factor receptor (EGFR). The proline-arginine motif PXXXPR in c-Cbl and SH3 domains of CIN85 are essential to this interaction. Here, we demonstrated that SH3KBP1-binding protein 1 (SHKBP1), which also contains two PXXXPR motifs, constitutively bound to SH3 domains of CIN85. Importantly, the binding of SHKBP1 prevented the interaction of CIN85 with c-Cbl and inhibited the translocation of CIN85 to EGFR-containing vesicles, thus reducing EGFR degradation and enhancing EGF-induced serum response element transcription activity. Therefore, our results indicated that SHKBP1 could promote EGFR signaling pathway by interrupting c-Cbl-CIN85 complex and inhibiting EGFR degradation. Copyright (C) 2011 John Wiley \& Sons, Ltd.
\end{abstract}

KEY WORDS-SH3KBP1-binding protein 1; Cbl-interacting protein of $85 \mathrm{kDa}$ (CIN85); c-Cbl; epidermal growth factor receptor; downregulation

\section{INTRODUCTION}

Epidermal growth factor receptor (EGFR) plays a critical role in the regulation of many biological responses such as cell proliferation, differentiation and cellular adhesion. ${ }^{1-4}$ Thus, the regulation of EGFR signaling is very important to maintain tissue homeostasis. ${ }^{5}$ Aberrations in the EGFR activity could lead to the development of various cancers such as lung, breast and brain cancers. ${ }^{3,6,7}$ To regulate the lifetime of this signaling, the activated EGFR also triggers the negative feedback loop that inhibits its activation by promoting its endocytosis and lysosomal degradation. Casitas B-lineage lymphoma (Cbl) family member $\mathrm{c}-\mathrm{Cbl}$ plays a crucial role in this process, acting as an ubiquitin ligase as well as an endocytic adaptor molecule. Upon growth factor stimulation, c-Cbl binds to activated EGFR and acts as a multifunctional adaptor protein to recruit additional endocytic regulatory proteins such as Cbl-interacting protein of 85 $\mathrm{kDa}$ (CIN85), which plays important roles in receptor endocytosis and degradation. ${ }^{7-9}$

Through the tyrosine kinase binding module and the ringfinger domain in its amino terminus, c-Cbl could promote the ubiquitination of various activated receptor tyrosine kinases

Supporting information may be found in the online version of this article. *Correspondence to: Kaixian Qian, College of lifescience, Zhejiang University, Hangzhou, Zhejiang, 310058, P. R. China. E-mail: qiankaixian@ hotmail.com; Jian-Guo Geng, Department of Biologic and Materials Sciences, University of Michigan School of Dentistry, Ann Arbor, MI 48109, USA. E-mail: jgeng@umich.edu.
(RTKs) such as EGFR and c-Met. ${ }^{10}$ The carboxyl terminus of c-Cbl contains a long proline-rich domain, which serves as an adaptor for binding to multiple signaling proteins and transducing intracellular signaling cascades. ${ }^{11}$ CIN85 is a ubiquitously expressed adaptor protein with three $\mathrm{SH} 3$ domains at the amino terminus, as well as a proline-rich region and a coiled-coil domain at the carboxyl terminus. ${ }^{12,13}$ The c-Cbl-CIN85 complex is formed through the interaction between proline-arginine motif PXXXPR (X denotes any amino acid) of c-Cbl and SH3 domains of CIN85. The arginine-to-alanine mutation in PXXXPR motif abolished the formation of the c-Cbl-CIN85 complex and attenuated the negative feedback regulation of EGFR signaling. ${ }^{14,15}$

Several recent findings indicated that EGFR signaling can be finely tuned by regulating the functions of c-Cbl-CIN85 complex. For example, Sprouty2 has been shown to sequester c-Cbl away from CIN85 and activated EGFR to inhibit the down-regulation of EGFR signaling. ${ }^{16-18}$ Another protein named cloned-out of library 1 (Cool 1), a nucleotide exchange factor for $\mathrm{Cdc} 42$, is able to form a complex with c-Cbl, thus preventing its interaction with activated EGFR and reducing EGFR degradation. ${ }^{18}$ Other proteins, such as infected cell protein 0 (ICP0) and Arf GTPase-activating proteins ASAP1, and adaptor proteins Huntingtin-interacting protein 1-related protein (Hip1R) and signal-transducing adaptor protein 1 (STAP1) also are implicated in the regulation of EGFR signaling. ${ }^{15,19}$

In the present study, we found SH3KBP1-binding protein 1 (SHKBP1) bound to SH3 domains of CIN85 via two 
PXXXPR motifs in its carboxyl terminus. SHKBP1 could compete with c-Cbl to interact with CIN85 and attenuate the down-regulation of EGFR signaling following receptor activation. Overexpression of SHKBP1 delayed the degradation of EGFR and enhanced EGFR signaling by disturbing the translocation of CIN85 to EGFR-containing vesicles. Taken together, these data provide a novel mechanism of the regulation of c-Cbl-CIN85 association and fine-tuning of EGFR activation.

\section{MATERIALS AND METHODS}

\section{Antibodies and plasmids}

EGF (PHG0311) was purchased from Invitrogen (Carlsbad, CA, USA). Rabbit anti-EGFR polyclonal antibody (06-129) and mouse anti-c-Cbl monoclonal antibody (05-440) were purchased from Millipore (Billerica, MA, USA). Mouse anti-FLAG monoclonal antibody (M2) (A2220) and mouse anti- $\alpha$-tubulin Clone B-5-1-2 (T5168) were purchased form Sigma (St. Louis, MO, USA). Mouse anti-Myc monoclonal antibody was prepared from a mouse hybridoma clone 9E10 obtained from ATCC (Manassas, VA, USA). Alexa fluor 594 goat anti-mouse $\operatorname{IgG}(\mathrm{H}+\mathrm{L})(\mathrm{A}-11005)$ and Alexa fluor 594 goat anti-rabbit $\operatorname{IgG}(\mathrm{H}+\mathrm{L})(\mathrm{A}-11012)$ were purchased from Invitrogen (Carlsbad, CA, USA). Full length cDNA of SHKBP1 (MMM1013-9201499) was purchased from Open Biosystems (Huntsville, AL, USA). Full-length cDNA of c-Cbl and CIN85 were kindly provided by Dr Biao Wang (Department of Medicine, University of Minnesota Medical School). EGFR/pRK5 and serum response element (SRE)-Luc reporter plasmids were kindly provided by Prof. Zhengjun Chen (Institute of Biochemistry and Cell Biology, Shanghai Institutes for Biological Sciences) and Dr Bernard Roizman (Department of Molecular Genetics and Cell Biology, University of Chicago), respectively. MycSHKBP1 and Myc-c-Cbl inserts were prepared by PCR using the corresponding full-length cDNA plasmids as template and cloned into the mammalian expression vector pCMV-3Tag2 (Stratagene, Santa Clara, CA, USA). Similarly, Flag-SHKBP1, Flag-CIN85, Flag-CIN85-3SH3 and Flag-CIN85-PCC PCR products were cloned into the pCMV-3Tag1 vector. GFPCIN85 was prepared by PCR using full-length CIN85 cDNA plasmid as template and cloned in to the pEGFPC1 vector. SHKBP1-R623A and SHKBP1-R684A were obtained by site-directed mutagenesis of wild-type SHKBP1 using the TaKaRa MutanBEST Kit (TaKaRa, Dalian, Liaoning, China). SHKBP1-R623A/R684A was obtained by site-directed mutagenesis of SHKBP1-R623A using the TaKaRa MutanBEST Kit. Sequences of all constructs were verified by sequencing, and the primers used for construction were listed in Table S1.

\section{Cells and transfection}

HEK293T and HeLa cells were purchased from ATCC (Manassas, VA, USA) and cultured in DMEM medium (Invitrogen, Carlsbad, CA, USA) supplemented with $10 \%$ fetal bovine serum (Gibco, Grand Island, NY, USA) and
$1 \%$ antibiotics (Sigma, St. Louis, MO, USA) in a humidified incubator at $37^{\circ} \mathrm{C}$ supplied with $5 \% \mathrm{CO}_{2}$. For transfection, $0.5 \times 10^{6}$ cells were seeded onto individual well of six-well plates and cultured for $24 \mathrm{~h}$ before transfection with DNA using LipofectAMINE reagent (Invitrogen, Carlsbad, CA, USA) following manufacturer's instructions. Thirty-six hours after transfection, the cells were incubated with serum-free DMEM medium for additional $12 \mathrm{~h}$ and subsequently treated with $100 \mathrm{ng} \cdot \mathrm{ml}^{-1}$ of EGF for the time indicated. After EGF treatment, the cells were washed with cold PBS and lysed in cold lysis buffer $\left(50 \mathrm{mmol} \cdot \mathrm{l}^{-1}\right.$ of $4-$ (2-hydroxyethyl)-1-piperazineethanesulfonic acid (HEPES), $\mathrm{pH} 7.4,150 \mathrm{mmol} \cdot \mathrm{l}^{-1}$ of $\mathrm{NaCl}, 1 \mathrm{mmol} \cdot \mathrm{l}^{-1}$ of EDTA, 1 mmol. $1^{-1}$ of EGTA, $10 \%$ glycerol and $1 \%$ Triton X-100) supplemented with a cocktail of protease and phosphatase inhibitors as previous reported. ${ }^{20}$

\section{Yeast two-hybrid experiment}

The ProQuest yeast two-hybrid system was used to study protein-protein interaction according to the manufacturer's instructions (Invitrogen, Carlsbad, CA, USA). DNA encoding SHKBP1 and its mutants were cloned into the pDBLeu vector, whereas DNA of CIN85 was cloned into the pPC86 vector. Yeast cells (MAV203 strain) were co-transformed with the CIN85 plasmid and either a wild type or mutant SHKBP1 plasmid to study protein-protein interaction. SHKBP1 or its mutants co-transformed with empty pPC86 and CIN85 co-transformed with empty pDBLeu were used as control. Meanwhile, co-transformed pPC86 and pDBLeu vectors were used as negative control in this experiment. After transformation, the yeasts were spread on the SCLeu-Trp plates (synthetic complete culture medium with $1.5 \%$ agar that lacks leucine and tryptophan prepared following the manufacturer's instruction) and placed in a $37^{\circ} \mathrm{C}$ incubator for 5-7 days. Visible yeast colonies were picked up and analysed using a LacZ beta-galactosidase assay with $\mathrm{X}$-gal substrate to determine the interaction of CIN85 with wild-type or mutant SHKBP1. The interaction was scored and compared with the positive and negative control yeast strains supplied by the manufacturer.

\section{Immuno-precipitation and Western blot}

Cell lysates were quantitated by Bio-Rad protein assay kit II (Bio-Rad Laboratories, Hercules, CA, USA). Same amount of protein from each lysate was mixed with either mouse anti-Myc or anti-FLAG (M2) monoclonal antibody and protein A beads (GE Healthcare, Piscataway, NJ, USA) in a microcentrifuge tube and placed on a rotator overnight at $4{ }^{\circ} \mathrm{C}$. Normal mouse IgG was used as control antibody for the immuno-precipitation. The immuno-complexes precipitated with protein A beads were washing in ice-cold lysis buffer a few times and finally resuspended and boiled in Uli Laemmli sample buffer. The immuno-precipitates were resolved using sodium dodecyl sulfate PAGE, transferred to Polyvinylidene Fluoride (PVDF) membranes and probed with the indicated primary antibodies as previously reported. ${ }^{21}$ 


\section{Immuno-fluorescence}

To study the co-localization of SHKBP1 or its mutants with CIN85, HEK293T cells seeded on collagen-coated cover slips were transfected with indicated expression plasmids for $36 \mathrm{~h}$ and starved for additional $12 \mathrm{~h}$. Transfected cells were then stimulated with or without EGF $\left(100 \mathrm{ng} \cdot \mathrm{ml}^{-1}\right.$ ) for $10 \mathrm{~min}$ and then fixed in $4 \%$ formaldehyde for $20 \mathrm{~min}$. After permeabilization and blocking, the cells were incubated with primary antibodies and then with Alexa Fluorconjugated secondary antibodies (Invitrogen, Carlsbad, CA, USA). The preparations were mounted, and the images were taken with a confocal microscope (Carl Zeiss, Jena, Germany).

\section{Luciferase reporter assay}

HEK293T or HeLa cells were seeded and cultured in collagencoated 12-well plates overnight. The cells were transfected with the indicated plasmids, together with SRE-Luc and beta-galactosidase reporter plasmids. Thirty-sixhours post-transfection, the cells were incubated with serum-free DMEM for an additional $12 \mathrm{~h}$ and then stimulated with 100 $\mathrm{ng} \cdot \mathrm{ml}^{-1} \mathrm{EGF}$ for the indicated time. Stimulated cells were lysed in $1 \times$ lysis buffer, and the lysates were mixed with luciferase assay reagent according to the manufacturer's instructions (Promega, Madison, MI, USA). After incubation in the dark for the indicated time, the luciferase activity was measured by a Victor Wallace luminometer (Turku, Finland), and the luciferase activity was normalized against beta-galactosidase activity measured in parallel. $^{22}$ The samples were prepared in triplicates and measured, and the normalized luciferase activity was expressed as the mean \pm SD.

\section{RESULTS}

\section{PXXXPR motifs of SHKBP1 constitutively bound to CIN85}

CIN85 was found to bind to proline-arginine motif PXXXPR contained in numerous adaptor proteins including c-Cbl, Cbl-b, Sprouty2, Cool1, ASAP1 and STAP1. ${ }^{2,15,17,18}$ Through bioinformatic analysis, we found that SHKBP1 had two PXXXPR motifs in the carboxyl terminus: PSPSPR (aa: 618-623) and PTPAPR (aa: 679-684), indicating potential interaction with CIN85. Indeed, yeast two-hybrid experiments confirmed the interaction of SHKBP1 with CIN85 (Table 1). The arginine residue within the prolinearginine motif has been shown to be essential for the interaction with $\mathrm{SH} 3$ domain, as the arginine-to-alanine mutation abolished this association. ${ }^{17}$ To explore the role of two PXXXPR motifs in the interaction of SHKBP1 with CIN85, arginine residues in these two motifs were mutated to alanine residues. Three SHKBP1 mutants-SHKBP1R623A (denoted as R623A), SHKBP1-R684A (denoted as R684A) and SHKPB1-R623A/R684A (denoted as R623A/ R684A) - were created and tested in the yeast two-hybrid interaction assay. Yeast colonies with R623A plasmid showed a strong positive signal, whereas colonies containing R684A plasmids only showed a weak positive signal in the LacZ assay (Table 1). In addition, colonies with the double mutant R623A/R684A plasmid showed no signal at all (Table 1). Taken together, these findings indicated that two PXXXPR motifs are responsible for the interaction of SHKBP1 with CIN85, although the contribution of the two motifs to the interaction seemed to be different.

To confirm that this interaction also occurs in mammalian cells, SHKBP1-expressing plasmid was co-transfected with CIN85-coding vector into HEK293T cells. Co-immunoprecipitation study demonstrated the interaction between SHKBP1 and CIN85 in the transfected cells (Figure 1a). Furthermore, SH3 domains (CIN85-3SH3) but not the prolinerich region and coiled-coil region (CIN85-PCC) contributed to the binding of CIN85 to SHKBP1 (Figure 1b). Although single mutants R623A and R684A could bind to CIN85, the double mutant R623A/R684A failed to interact with CIN85 (Figure 1c). Interestingly, we found that the interaction of SHKBP1 with CIN85 was not affected by EGF stimulation (Figure 1c). The above data suggested a direct and constitutive interaction between SHKBP1 and CIN85, which was mediated by PXXXPR motifs of SHKBP1.

\section{SHKBP1 competed with c-Cbl to bind to CIN85 through its two PXXXPR motifs}

It was known that c-Cbl associated with CIN85 through the PXXXPR motif of c-Cbl and the SH3 domain(s) of CIN85, and EGF stimulation could further promote the assembly of

Table 1. Yeast two-hybrid experiment examining the interaction of SHKBP1 or its mutants with CIN85

\begin{tabular}{|c|c|c|c|c|c|}
\hline & SHKBP1 & SHKBP1-R623A & SHKBP1-R684A & SHKBP1-R623A/684A & pDBLeu \\
\hline Plasmids & CIN85 & CIN85 & CIN85 & CIN85 & pPC86 \\
\hline LacZ activity & +++ & +++ & + & - & - \\
\hline Plasmids & $\left\{\begin{array}{l}\text { SHKBP1 } \\
\text { pPC } 86\end{array}\right.$ & $\begin{array}{l}\text { SHKBP1-R623A } \\
\text { pPC86 }\end{array}$ & $\begin{array}{l}\text { SHKBP1-R684A } \\
\text { pPC86 }\end{array}$ & $\begin{array}{l}\text { SHKBP1- R623A/684A } \\
\text { pPC86 }\end{array}$ & $\begin{array}{l}\text { pDBLeu } \\
\text { CIN85 }\end{array}$ \\
\hline LacZ activity & - & - & - & - & - \\
\hline
\end{tabular}

MAV203 yeast strain was co-transformed with SHKBP1/pDBLeu or its mutants plasmids and CIN85/pPC86 plasmids. SHKBP1 or its mutants co-transformed with empty pPC86 and CIN85 co-transformed with empty pDBLeu were used as control. pPC86 and pDBLeu vectors were used as negative control. Transformed yeasts were selected in SC-Leu-Trp plates, and a LacZ assay was performed with colonies grown on the SC-Leu-Trp plates and control yeast strains: a strong positive control A, a weak positive control C and a negative control supplied by manufacturer. ('+++' indicates a strong positive signal, '+' indicates a weak positive signal and '-' indicates a negative signal). 
a

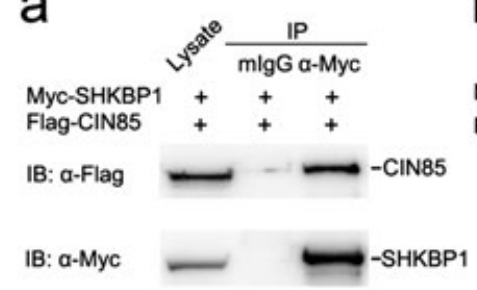

b

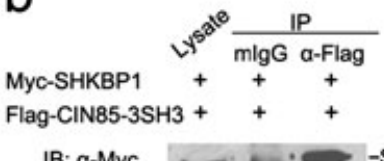

IB: a-Myc

- ba: -SHKBP1

IB: a-Flag

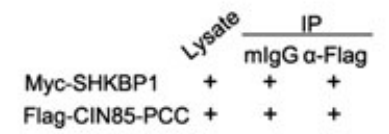

IB: a-Myc - -SHKBP1

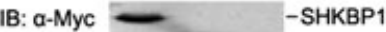

-CIN85-3SH3 IB: a-Flag

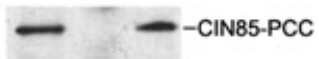

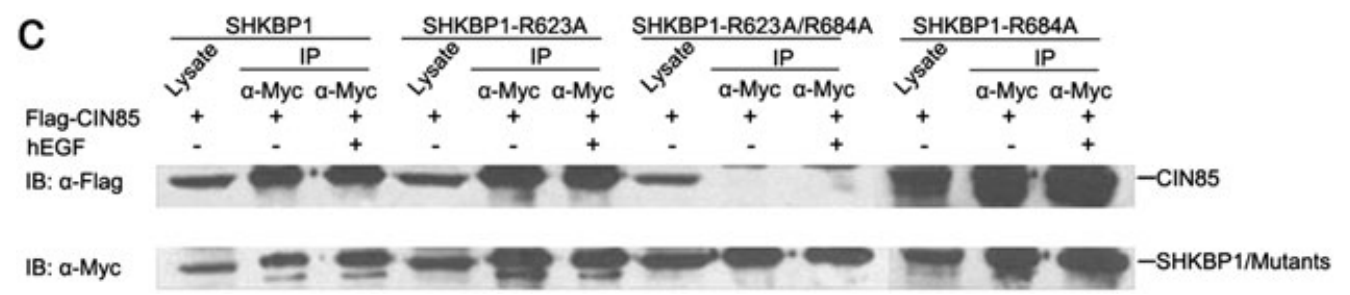

Figure 1. PXXXPR motifs of SHKBP1 constitutively bound to CIN85. (a) HEK293T cells co-transfected with Myc-SHKBP1 and Flag-CIN85 plasmids were lysed $48 \mathrm{~h}$ after transfection. Immuno-precipitations were performed with anti-Myc antibody and control mouse IgG (mIgG). Immuno-precipitated proteins were processed for Western blot with anti-FLAG antibody. The membrane was stripped and re-blotted with anti-Myc antibody. (b) HEK293T cells co-transfected with Flag-CIN85-3SH3 or Flag-CIN85-PCC and Myc-SHKBP1 plasmids were lysed 48h after transfection, and cell lysates were immunoprecipitated with anti-FLAG antibody and control mIgG. Immuno-precipitated proteins were processed for Western blot with anti-Myc antibody. The membrane was stripped and re-blotted with anti-FLAG antibody. (c) HEK293T cells transfected with Myc-SHKBP1 or its mutants and Flag-CIN85 plasmids were starved in serum-free DMEM for $12 \mathrm{~h}$ and then treated with $100 \mathrm{ng} \cdot \mathrm{ml}^{-1}$ of recombinant human EGF for $10 \mathrm{~min}$. The cells were lysed and immuno-precipitated with antiMyc antibody. Immuno-precipitated proteins were processed for Western blot with anti-FLAG antibody, and the membrane was then stripped and re-blotted with anti-Myc antibody

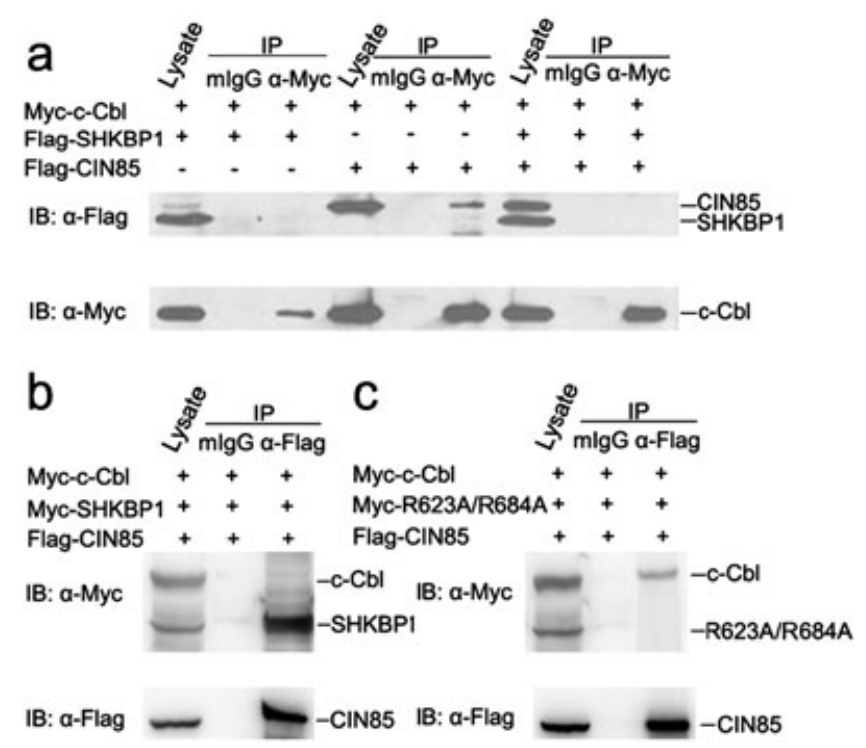

Figure 2. SHKBP1 competed with c-Cbl to bind to CIN85 through its two PXXXPR motifs. (a) HEK293T cells co-transfected with expression plasmids encoding Myc-c-Cbl, Flag-SHKBP1 or Flag-CIN85 were lysed 48h after transfection, and cell lysates were immuno-precipitated with anti-Myc antibody and control mIgG. Immuno-precipitated proteins were processed for Western blot with anti-FLAG antibody, and the membrane was stripped and re-blotted with anti-Myc antibody. (b) HEK293T cells co-transfected with plasmids encoding Myc-c-Cbl, Myc-SHKBP1 and Flag-CIN85 were lysed 48h after transfection, and cell lysates were immuno-precipitated with anti-FLAG antibody and control mIgG. Immuno-precipitated proteins were processed for Western blot with anti-Myc antibody, and the membrane was stripped and re-blotted with anti-FLAG antibody. (c) HEK293T cells co-transfected with plasmids encoding Myc-c-Cbl, Myc-R623A/684A and Flag-CIN85 were lysed 48h after transfection, and cell lysates were immuno-precipitated with anti-FLAG antibody and control mIgG. Immuno-precipitated proteins were processed for Western blot with anti-Myc antibody, and the membrane was stripped and re-blotted with anti-FLAG antibody

this complex. ${ }^{4,9}$ The dependence of the SHKBP1-CIN85 interaction on PXXXPR motifs of SHKBP1 led us to assume whether SHKBP1 could interfere with the association between $\mathrm{c}-\mathrm{Cbl}$ and CIN85. As previously reported, c-Cbl could bind to CIN85 (Figure 2a). However, such an association was completely abolished in the presence of ectopically 
expressed SHKBP1 (Figure 2a). c-Cbl did not bind to SHKBP1 and failed to affect the association of SHKBP1 and CIN85 in co-transfected cells (Figures 2a and 2b). Importantly, the double mutant R623A/R684A failed to affect the interaction between $\mathrm{c}-\mathrm{Cbl}$ and CIN85 (Figure 2c). These data indicated that SHKBP1 interfered with the formation of the c-Cbl-CIN85 complex through sequestration of CIN85 via its two PXXXPR motifs.

\section{SHKBPI inhibited EGFR degradation after EGF stimulation}

Next, we hypothesized that the direct binding of SHKBP1 to CIN85 could attenuate c-Cbl-CIN85 complex promoted EGFR degradation. ${ }^{4}$ To test it, the EGFR level in the transfected cells was measured following EGF stimulation of cells transected with wild-type or various mutants of SHKBP1. As shown in Figure 3a, the EGFR level was upregulated immediately upon EGF stimulation and then reduced gradually. The EGFR protein was not detectable about 180 min after EGF stimulation in mock vector transfected cells (Figure 3a). Overexpression of wild-type SHKBP1 and R623A mutant strongly delayed the degradation of EGFR upon EGF activation (Figures $3 b$ and $3 c$ ). In contrast, the receptor degradation was only slightly delayed by the overexpression of R684A mutant (Figure 3d), whereas the R623A/R684A double mutant showed no effect at all (Figure 3e).
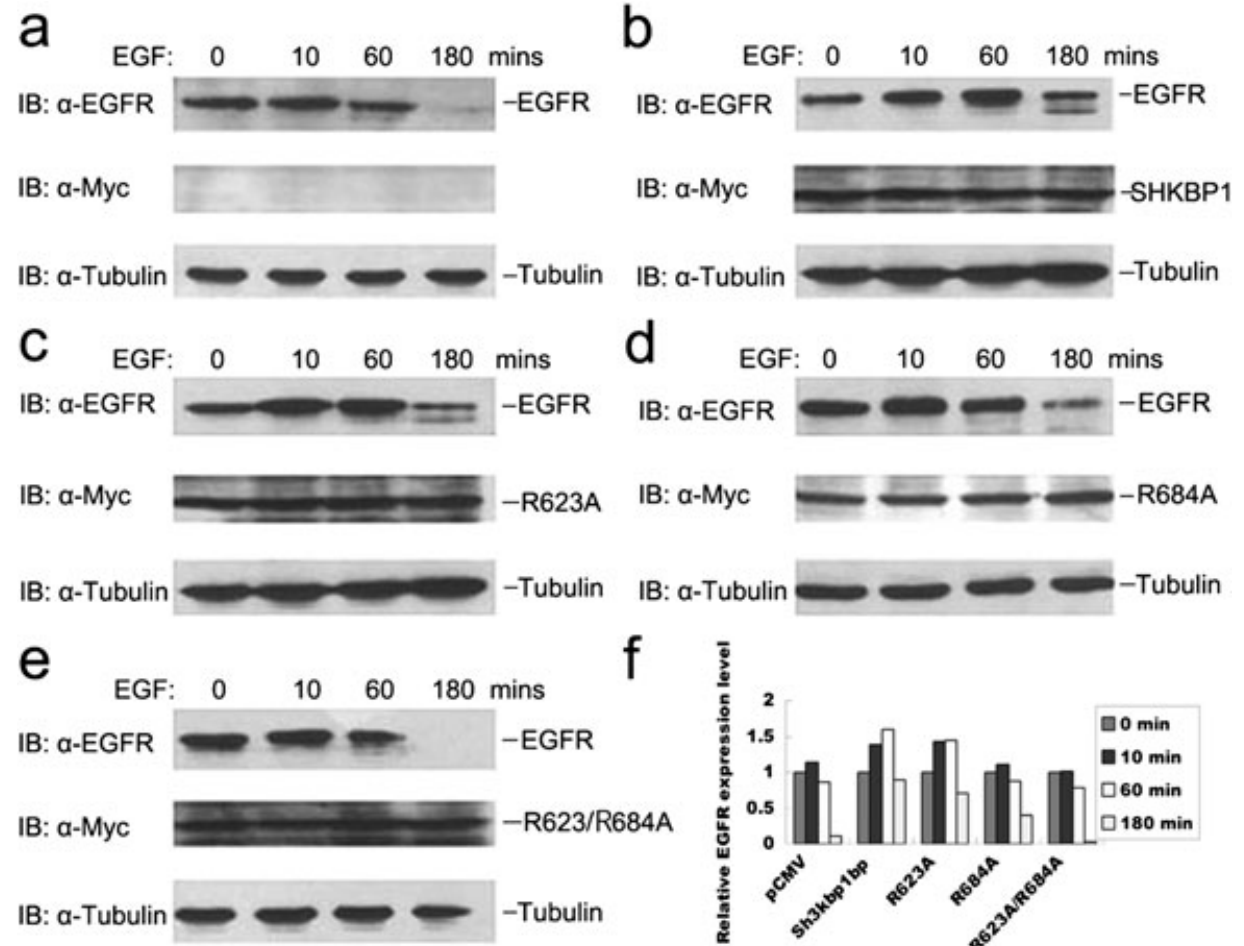

\section{SHKBP1 disturbed the translocation of CIN85 to EGFR-containing vesicles}

CIN85 was found to co-localize with EGFR and c-Cbl complex upon EGF stimulation, which is important to receptor endocytosis and the formation of EGFR-containing vesicles during EGFR degradation. ${ }^{8,23}$ We therefore explored the localization of SHKBP1 and its role on CIN85 translocation after EGF stimulation by immuno-fluorescence. CIN85 translocated to EGFR-containing vesicles upon EGF stimulation (Figures $4 \mathrm{a}$ and $4 \mathrm{~d}$ ). Overexpression of wild-type SHKBP1 and single mutant R623A and R684A inhibited the formation of EGFR-containing vesicles and the translocation of CIN85 (Figures 4b, 4e and Figure S1). In contrast, the expression of double mutant R623A/R684A did not disturb the translocation of CIN85 to EGFR-containing vesicles (Figures $4 \mathrm{c}$ and $4 \mathrm{f}$ ). These data suggested that SHKBP1 could disturb the translocation of CIN85 to

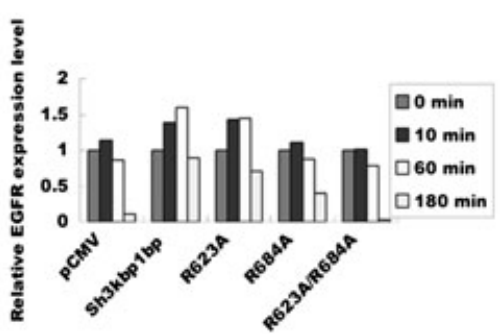

Figure 3. SHKBP1 inhibited EGFR degradation after EGF stimulation. HeLa cells were transfected with indicated expression plasmids: pCMV (a), MycSHKBP1 (b), Myc-R623A (c), Myc-R684A (d) and Myc-R623A/684A (e), respectively. Thirty-sixhours post-transfection, cells were starved in serum-free DMEM for $12 \mathrm{~h}$. The cells were then stimulated with $100 \mathrm{ng} \cdot \mathrm{ml}^{-1}$ of EGF for the indicated times. Lysates were prepared and analysed using Western blot with anti-EGFR, anti-Myc or anti- $\alpha$-tubulin antibodies. The relative level of EGFR (a-e) was quantified using densitometry scan and graphed in (f) 
a
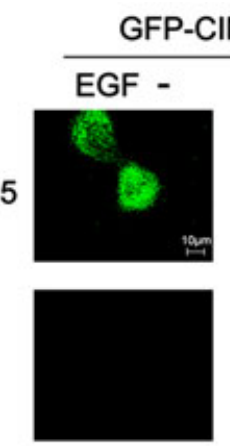

Anti-Myc

GFP-CIN85

$\frac{\mathrm{IN85}+\text { pCMV }}{\text { EGF + }}$
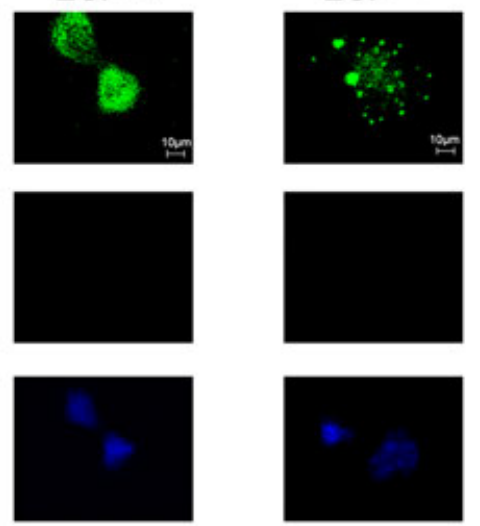

Merge
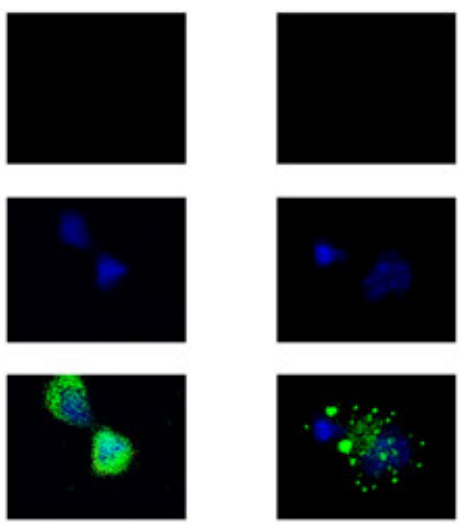

d
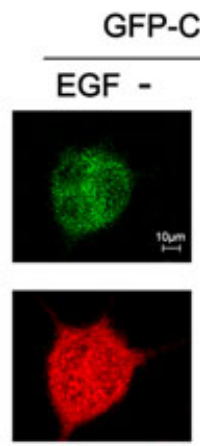

DAPI

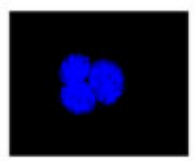

Merge

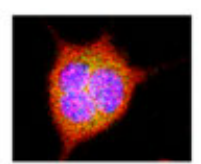

b
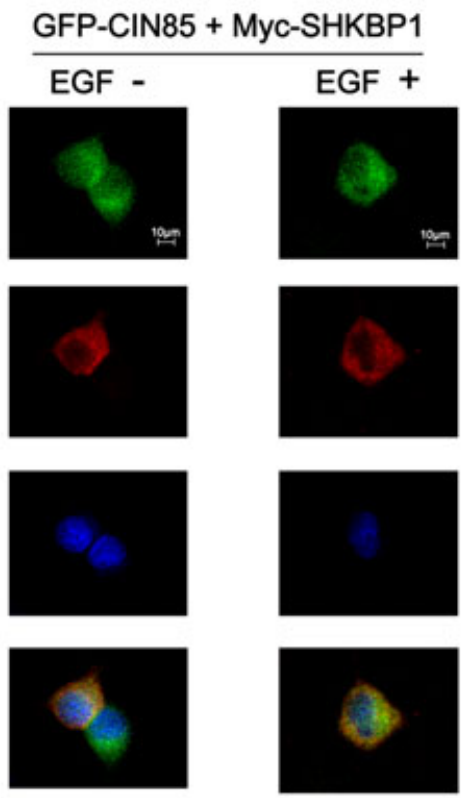

e
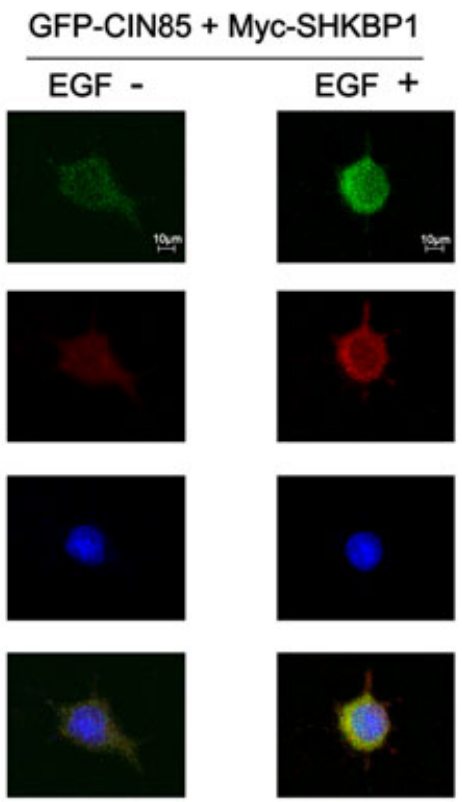

C GFP-CIN85 + Myc-R623A/R684A
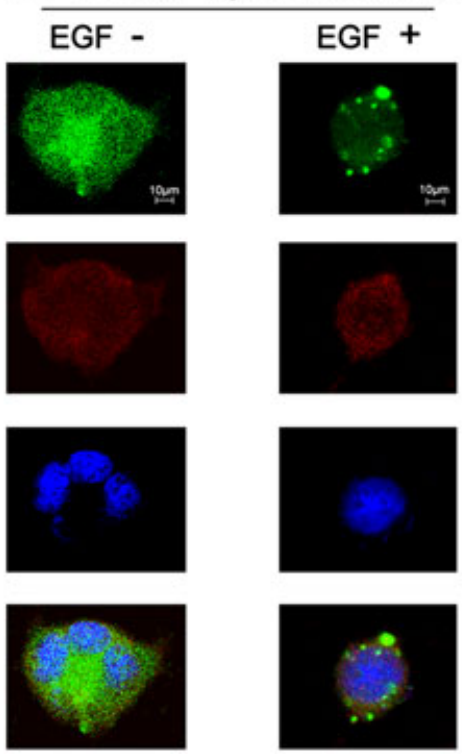

f GFP-CIN85 + Myc-R623A/R684A
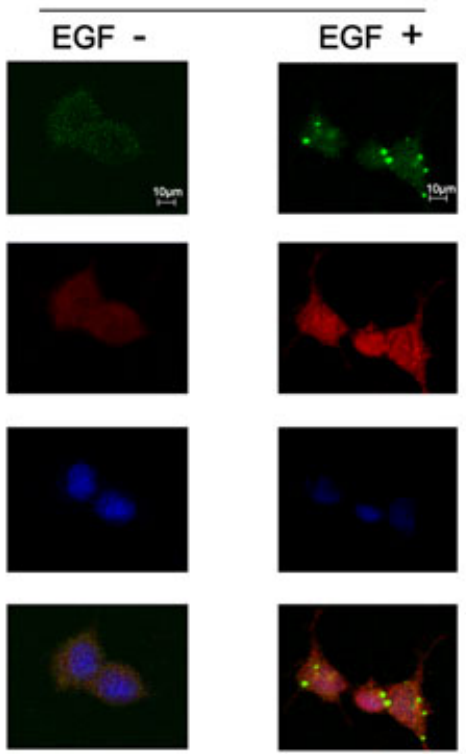

Figure 4. SHKBP1 disturbed the translocation of CIN85 to EGFR degradation vesicles. HEK293T cells were co-transfected with GFP-CIN85 plasmid and control pCMV mock vector (a, d), Myc-SHKBP1 (b, e) or Myc-R623A/R684A (c, f). Thirty-sixhours post-transfection, cells were starved in serum-free DMEM for $12 \mathrm{~h}$. The cells were left unstimulated or stimulated with hEGF for $10 \mathrm{~min}$. Immuno-fluorescence studies on cells were performed with antibodies against Myc tag $(a, b, c)$ or EGFR (d, e, f), and the nucleus was stained with DAPI. The merged pictures were shown in the bottom panel, and the staff $(10 \mu \mathrm{m})$ was shown in the upper panel

EGFR-containing vesicles upon EGF stimulation via the interaction of its PXXXPR motifs with CIN85.

\section{SHKBP1 enhanced EGF-induced SRE transcription activity}

Finally, we examined whether the reduction in EGFR degradation caused by overexpression of SHKBP1 would lead to the alteration in the downstream signaling. It was known that activated EGFR can induce transcription activity of promoters with the SRE. ${ }^{19}$ Therefore, the SRE luciferase reporter assay was used to examine the effect of SHKBP1 on EGFR signaling. Different amounts of SHKBP1 were co-transfected with the SRE-Luc gene reporter, c-Cbl, CIN85 and EGFR into HEK293T cells, and the luciferase 
activities in transfected cells were measured. The luciferase activities showed a dose-dependent effect of SHKBP1 on SRE transcription (Figure S2). Moreover, overexpression of SHKBP1, R623A or R684A mutant could significantly increase the SRE transcription activity by threefold $(P=0.001), 2 \cdot 5$-fold $(P=0.003)$ and $2 \cdot 6$-fold $(P=0.002)$, when compared with the mock vector group, respectively (Figure 5a). Similarly, overexpression of SHKBP1, R623A or R684A mutants in HeLa cells could increase the SRE transcription activity by 2.2 fold $(P=0.003), 1.7$ fold $(P=0.036)$ and $2 \cdot 0$ fold $(P=0 \cdot 001)$, respectively (Figure $5 \mathrm{~b})$. However, R623A/R684A double mutant had little effect on SRE-Luc reporter transcription in both HEK293T and HeLa
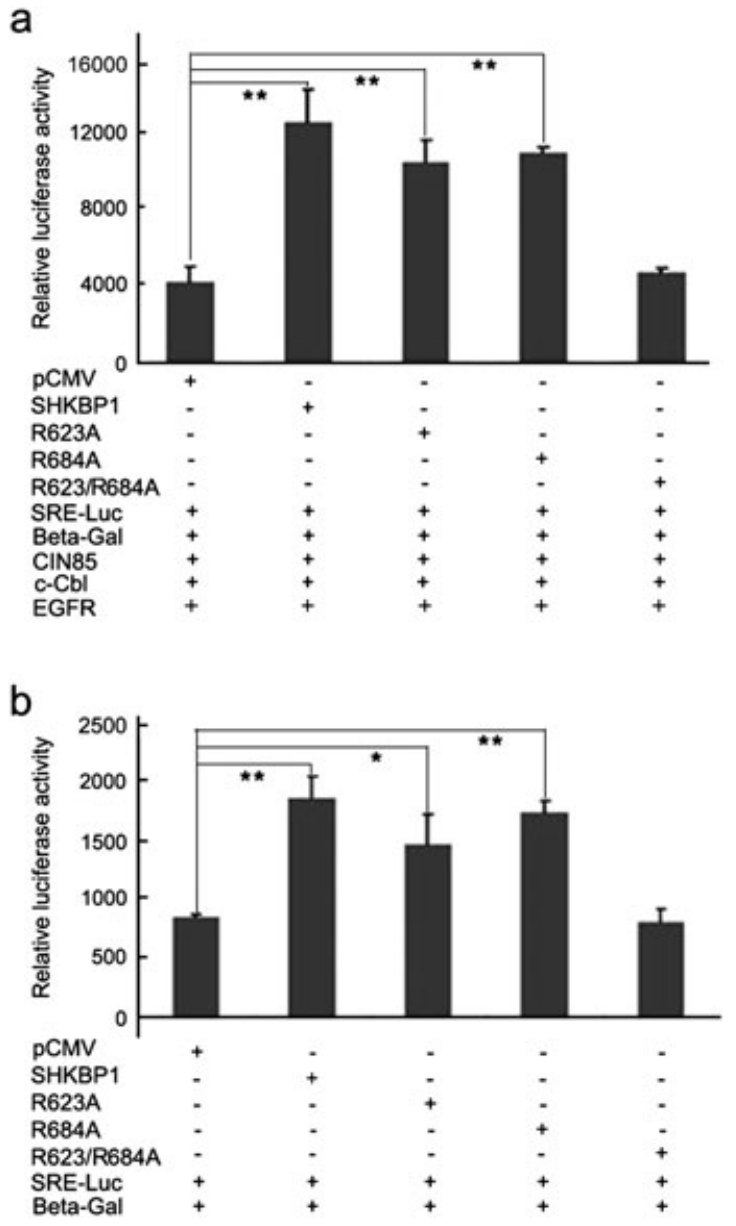

Figure 5. SHKBP1 enhanced EGF-induced SRE transcription activity. (a) HEK293T cells were co-transfected with EGFR (100ng), c-Cbl (100ng), CIN85 (100ng), SRE-Luc (100ng), $\beta$-gal (50ng) and SHKBP1 or its mutants plasmids (300ng). Thirty-sixhours post-transfection, cells were starved in serum-free DMEM for $12 \mathrm{~h}$. The cells were then stimulated with EGF for another $12 \mathrm{~h}$ and processed for luciferase assay. (b) HeLa cells were co-transfected with SRE-Luc (200ng), $\beta$-gal (100ng) and SHKBP1 or its mutants plasmids (300ng). Thirty-sixhours post-transfection, cells were starved in serum-free DMEM for $12 \mathrm{~h}$. The cells were then stimulated with EGF for additional $12 \mathrm{~h}$ and processed for luciferase assay. The luciferase activity was measured in triplicated samples and expressed as the mean \pm SD. Student's $t$-test was performed to compare the statistical difference between each group to pCMV control group. $* P<0.05 ; * * P<0 \cdot 01$ cells, indicating that SHKBP1 could enhance EGFR signaling through its interaction with CIN85.

\section{DISCUSSION}

In the current study, we found that SHKBP1 could bind to SH3 domains of CIN85 through its PXXXPR motifs in the carboxyl terminus (Figure 1 and Table 1). We further showed that the interaction of SHKBP1 with CIN85 could abolish c-Cbl-CIN85 complex formation (Figure 2), thus retarding EGFR degradation by disturbing the translocation of CIN85 to EGFR-containing vesicles following EGF stimulation (Figures 3 and 4 and Figure S1). Consistent with these observations, SHKBP1-enhanced EGFR signaling induced the SRE transcriptional reporter activity, which required at least one intact PXXXPR motif (Figure 5 and Figure S2).

The binding of EGF to EGFR induces receptor activation and downstream signaling cascades important to cell proliferation, differentiation and migration. ${ }^{1,2,4}$ Aberrant activation or overexpression of EGFR is commonly found in various cancers. It is clear that proper regulation of EGFR signaling is essential for normal physiology. ${ }^{1,6}$ The c-CblCIN85 complex has been shown to play a critical role in the down-regulation of activated EGFR. c-Cbl is associated with the cytoplasmic domain of EGFR upon receptor activation and catalyses the ubiquitination of EGFR. ${ }^{7}$ c-Cbl acts as a multifunctional adaptor protein to recruit CIN85 to the EGFR-c-Cbl complex, which facilitates the endocytosis of ubiquitinated EGFR and subsequent degradation. Therefore, the perturbation of EGFR-c-Cbl-CIN85 complex could have severe consequences in EGFR-regulated cell growth control. This regulatory mechanism has been reported for many c-Cbl-associated proteins. For example, Sprouty 2 not only acts at the interface of the c-Cbl-CIN85 complex but also directly associates with $\mathrm{c}-\mathrm{Cbl}$ to sequester $\mathrm{c}-\mathrm{Cbl}$ from activated EGFR, thus inhibiting the down-regulation of EGFR signaling. ${ }^{16,17}$ Additionally, Cool-1, a nucleotide exchange factor for $\mathrm{Cdc} 42$, is able to form a complex with c-Cbl, thereby sequestering c-Cbl from activated EGFR and preventing the down-regulation of EGFR signaling. ${ }^{18}$

We found SHKBP1 as a new regulator in EGFR activation. Unlike Sprouty2 and Cool-1 that promote EGFR signaling through their interaction with $\mathrm{c}-\mathrm{Cbl}$, SHKBP1 could retard EGFR degradation and up-regulate EGFR signaling by binding to CIN85 and sequestering CIN85 from c-CblCIN85 complex. PXXXPR motifs were essential for this function of SHKBP1. Therefore, our results may reveal a new mechanism of enhanced EGFR signaling in the development of various diseases such as cancers. It would be interesting to investigate whether the interaction of SHKBP1 with CIN85 also is involved in the regulation of other RTKs during cancer development.

In summary, our study demonstrated that SHKBP1 inhibited c-Cbl-CIN85 complex-mediated down-regulation of EGFR signaling by sequestration of CIN85. This novel mechanism provides an alternative route for the development of drugs targeting the EGFR signaling pathway. 


\section{CONFLICT OF INTEREST}

The authors have declared that there is no conflict of interest.

\section{ACKNOWLEDGEMENTS}

We thank Dr Biao Wang, Prof. Zhengjun Chen and Dr Bernard Roizman for providing cDNAs and constructs, and Dr Qiu Chen for giving advice.

\section{REFERENCES}

1. Dikic I, Giordano S. Negative receptor signalling. Curr Opin Cell Biol 2003; 15: 128-135. doi:10.1016/S0955-0674(03)00004-8.

2. Haglund K, Shimokawa N, Szymkiewicz I, Dikic I. Cbl-directed monoubiquitination of CIN85 is involved in regulation of ligandinduced degradation of EGF receptors. Proc Natl Acad Sci USA 2002; 99: 12191-12196. doi:10.1073/pnas.192462299.

3. Schneider MR, Sibilia M, Erben RG. The EGFR network in bone biology and pathology. Trends Endocrinol Metab 2009; 20: 517-524. doi:10.1016/j.tem.2009.06.008.

4. Soubeyran P, Kowanetz K, Szymkiewicz I, Langdon WY, Dikic I. Cbl-CIN85-endophilin complex mediates ligand-induced downregulation of EGF receptors. Nature 2002; 416: 183-187. doi:10.1038/ 416183a.

5. Waterman H, Yarden Y. Molecular mechanisms underlying endocytosis and sorting of ErbB receptor tyrosine kinases. FEBS Lett 2001; 490: 142-152. doi:10.1016/S0014-5793(01)02117-2.

6. Saif MW. Colorectal cancer in review: the role of the EGFR pathway. Expert Opin Investig Drugs 2010; 19: 357-369. doi:10.1517/ 13543781003593962

7. Thien CB, Langdon WY. Cbl: many adaptations to regulate protein tyrosine kinases. Nat Rev Mol Cell Biol 2001; 2: 294-307. doi:10.1038/ 35067100 .

8. Szymkiewicz I, Kowanetz K, Soubeyran P, Dinarina A, Lipkowitz S, Dikic I. CIN85 participates in Cbl-b-mediated down-regulation of receptor tyrosine kinases. J Biol Chem 2002; 277: 39666-39672. doi:10.1074/jbc.M205535200.

9. Pennock S, Wang Z. A tale of two Cbls: interplay of c-Cbl and Cbl-b in epidermal growth factor receptor downregulation. Mol Cell Biol 2008; 28: 3020-3037. doi:10.1128/MCB.01809-07.

10. Tan YH, Krishnaswamy S, Nandi S, et al. CBL is frequently altered in lung cancers: its relationship to mutations in MET and EGFR tyrosine kinases. PLoS One 2010; 5: e8972. doi:10.1371/journal.pone.0008972.
11. Jozic D, Cardenes N, Deribe YL, Moncalian G, Hoeller D, Groemping Y, Dikic I, Rittinger K, Bravo J. Cbl promotes clustering of endocytic adaptor proteins. Nat Struct Mol Biol 2005; 12: 972-979. doi:10.1038/ nsmb1000.

12. Take H, Watanabe S, Takeda K, Yu ZX, Iwata N, Kajigaya S. Cloning and characterization of a novel adaptor protein, CIN85, that interacts with c-Cbl. Biochem Biophys Res Commun 2000; 268: 321-328. doi:10.1006/bbrc.2000.2147.

13. Watanabe S, Take H, Takeda K, Yu ZX, Iwata N, Kajigaya S. Characterization of the CIN85 adaptor protein and identification of components involved in CIN85 complexes. Biochem Biophys Res Commun 2000; 278: 167-174. doi:10.1006/bbrc.2000.3760S0006291X(00)93760-4

14. Dikic I. CIN85/CMS family of adaptor molecules. FEBS Lett 2002; 529: 110-115. doi:10.1016/S0014-5793(02)03188-5.

15. Kowanetz K, Husnjak K, Holler D, et al. CIN85 associates with multiple effectors controlling intracellular trafficking of epidermal growth factor receptors. Mol Biol Cell 2004; 15: 3155-3166. doi:10.1091/ mbc.E03-09-0683.

16. Hall AB, Jura N, DaSilva J, Jang YJ, Gong D, Bar-Sagi D. hSpry2 is targeted to the ubiquitin-dependent proteasome pathway by cCbl. Curr Biol 2003; 13: 308-314. doi:10.1016/S0960-9822(03) 00086-1.

17. Haglund K, Schmidt MH, Wong ES, Guy GR, Dikic I. Sprouty2 acts at the $\mathrm{Cbl} / \mathrm{CIN} 85$ interface to inhibit epidermal growth factor receptor downregulation. EMBO Rep 2005; 6: 635-641. doi:10.1038/sj.embor. 7400453.

18. Feng Q, Baird D, Peng X, Wang J, Ly T, Guan JL, Cerione RA. Cool-1 functions as an essential regulatory node for EGF receptor- and Srcmediated cell growth. Nat Cell Biol 2006; 8: 945-956. doi:10.1038/ ncb1453.

19. Liang Y, Kurakin A, Roizman B. Herpes simplex virus 1 infected cell protein 0 forms a complex with CIN85 and Cbl and mediates the degradation of EGF receptor from cell surfaces. Proc Natl Acad Sci USA 2005; 102: 5838-5843. doi:10.1073/pnas.0501253102.

20. Wang HB, Wang JT, Zhang L, et al. P-selectin primes leukocyte integrin activation during inflammation. Nat Immunol 2007; 8: 882-892. doi:10.1038/ni1491.

21. Wang B, Xiao Y, Ding BB, et al. Induction of tumor angiogenesis by Slit-Robo signaling and inhibition of cancer growth by blocking Robo activity. Cancer Cell 2003; 4: 19-29. doi:10.1016/S1535-6108(03) 00164-8.

22. Criqui-Filipe P, Ducret C, Maira SM, Wasylyk B. Net, a negative Ras-switchable TCF, contains a second inhibition domain, the CID, that mediates repression through interactions with $\mathrm{CtBP}$ and de-acetylation. EMBO J 1999; 18: 3392-3403. doi:10.1093/ emboj/18.12.3392.

23. Zhang J, Zheng X, Yang X, Liao K. CIN85 associates with endosomal membrane and binds phosphatidic acid. Cell Res 2009; 19: 733-746. doi:10.1038/cr.2009.51. 